\title{
Phase transition in Schwarzschild-de Sitter spacetime
}

\author{
D.Momeni* \\ Department of Physics ,Faculty of science, \\ Islamic Azad University, Karaj Branch. .Iran, \\ Karaj, Rajaei shahr, P.O.Box: 31485-313 \\ A.Azadit \\ Department of Physics, University of Tehran, \\ North Karegar Ave., 14395-547, Teheran, Iran
}

\begin{abstract}
Using a static massive spherically symmetric scalar field coupled to gravity in the Schwarzschild-de Sitter (SdS) background, first we consider some asymptotic solutions near horizon and their local equations of state(E.O.S) on them. We show that near cosmological and event horizons our scalar field behaves as a dust. At the next step near two pure de-Sitter or Schwarzschild horizons we obtain a coupling dependent pressure to energy density ratio. In the case of a minimally couplling this ratio is -1 which springs to the mind thermodynamical behavior of dark energy. If having a negative pressure behavior near these horizons we concluded that the coupling constant must be $\xi<\frac{1}{4}$. Therefore we derive a new constraint on the value of our coupling $\xi$. These two different behaviors of unique matter in the distinct regions of spacetime at present era can be interpreted as a phase transition from dark matter to dark energy in the cosmic scales and construct a unified scenario.
\end{abstract}

PACS numbers: $98.80 .-\mathrm{k}, 95.35 .+\mathrm{d}, 95.36 .+\mathrm{x}$

\section{INTRODUCTION}

After many years of considering that the Universe is filled with a dark matter and with a dark energy causing an acceleration of the expansion of the Universe, we have still no direct evidence of their existences. In addition, observations of supernova of type Ia tend to cause trouble to common dark energy models [1], and open the way to new kind of models to explain the observed repulsing effects [2]. Alternative models has created to explain the cosmological observations, and in particular some of them try to solve the dark energy and dark matter problems by unified object in which the dark matter and the dark energy are in fact different aspects of a same fluid, the "dark fluid". For instance mentioned that the Generalized Chaplygin Gas model [3] follows this idea and is presently under scrutiny. .As scalar field-based models for dark energy and dark matter exist in the literature [4], it seems interesting to investigate unifying dark fluid models based on scalar fields. This idea has been proposed in [5].

On the other hand spherical symmetric solutions of a scalar field in static spacetimes is the most simple case to study [6]. We know that there is no simple exact solution for a static massive scalar field as a source of gravity. Some attempts to construct such model were failed because of mathematically complications. There is only a massless minimally coupled scalar field version of gravity [7]. If we insert mass or coupling constant, equation 
can not be integrated in closed forms. Considering unverse acceleration, if we assume that our universe be asymptotically de Sitter and there exists a massive black hole in the center of galaxy,for the metric we can write a simple schwarzschild-de Sitter line element which in weak field approximation asymptotically leads to de Sitter.There is some significant works on the SdS black hole from stability viewpoint of higher dimensional spacetimes [9] and also on calculation of quasinormal modes of four dimensional SdS black holes [10]. In this work we present a massive-coupled-scalar-field in the SdS background and show that in the scale of our cosmos, dust and negative pressure matter are the same and essentially can be unified in present era as a negative pressure matter that evolves like a dust near the horizons.

\section{SDS GEOMETRY}

SdS space time is a well known vacuum solution of Einstein field equations with a constant curvature $R=+\frac{12}{a^{2}}$ where $a$ is related to Cosmological-Constant through $\Lambda=\frac{3}{a^{2}}$. It's geometry as a 4-dim Riemmanian manifold can be represented as:

$$
d s^{2}=-f(r) d t^{2}+1 / f(r) d r^{2}+r^{2}\left(d \theta^{2}+\sin ^{2} \theta d \varphi^{2}\right)
$$

Where $f(r)=1-\frac{2 M}{r}-\frac{r^{2}}{a^{2}}$. Depending on the values of $9 M^{2} \Lambda$ where $M$ is black-hole (B.H.) mass we have three distinct cases:

1. If $9 M^{2} \Lambda=1$ we have an extremal B.H. In this case both cosmological and event horizons coincide. That is $r_{+}=r_{c}$.

2. If $9 M^{2} \Lambda>1$ We've no B.H. solution .

3. If $0<9 M^{2} \Lambda<1$ we have Black hole solution with two positive horizons as cosmological (which is denoted by $r_{c}$ ) and event horizons $r_{+}$and an un-physical negative horizon as: $r_{n}=-\left(r_{c}+r_{+}\right)$. In this work we only restricted ourselves to Black hole case.

\section{FIELD EQS}

A simple coupled massive scalar field in a curved space-time is represented by a Lagrangian [7]:

$$
L=\frac{1}{2}\left(\phi_{; \mu} \phi^{; \mu}-\xi R \phi^{2}-m^{2} \phi^{2}\right)
$$

Matter action is:

$$
S_{\text {matter }}=\int \sqrt{-g} \frac{1}{2}\left(\phi_{; \mu} \phi^{; \mu}-\xi R \phi^{2}-m^{2} \phi^{2}\right) d^{4} x
$$

Related Energy-Momentum tensor and equation of motion(E.O.M) for field are [8] :

$$
\begin{array}{r}
T_{\mu \nu}=(1-2 \xi) \phi_{; \mu} \phi_{; \nu}+\left(2 \xi-\frac{1}{2}\right) g_{\mu \nu}\left(g^{\rho \sigma} \phi_{; \rho} \phi_{; \sigma}\right) \\
-2 \xi \phi_{; \mu \nu} \phi+\gamma g_{\mu \nu} \phi^{2} \\
\nabla_{\mu} \nabla^{\mu} \phi+\left(m^{2}+\xi R\right) \phi=0
\end{array}
$$

In a SdS space time we denote $\gamma$ :

$$
\gamma=m^{2} \frac{1-4 \xi}{2}-\frac{3 \xi(8 \xi+1)}{a^{2}}
$$


Where $\mathrm{m}$ is our scalar field mass. For a SdS B.H we can take scalar field as a function of radial coordinate $r$ to preserve isotropy of spacetime. Also we can ignore from all back reaction effects in this model. The energy density and pressure are:

$$
\begin{array}{r}
\rho=g^{00} T_{00}=f(r)\left(2 \xi-\frac{1}{2}\right)\left(\phi^{\prime}\right)^{2}-\xi f(r)^{\prime} \phi \phi^{\prime}+\gamma \phi^{2} \\
P_{r}=g^{r r} T_{r r}= \\
\frac{1}{2} f(r)\left(\phi^{\prime}\right)^{2}-2 \xi \phi\left(f(r) \phi^{\prime \prime}\right. \\
\left.+\frac{1}{2}(f(r))^{\prime} \phi^{\prime}\right)+\gamma \phi^{2}
\end{array}
$$

For a massless-minimally coupled scalar field our expressions for radial pressure and energy density become very simple :

$$
\begin{gathered}
\rho=-\frac{1}{2} f(r)\left(\phi^{\prime}\right)^{2} \\
P_{r}=\frac{1}{2} f(r)\left(\phi^{\prime}\right)^{2}
\end{gathered}
$$

Also for a massive minimally coupled model we have :

$$
\begin{gathered}
\rho=-\frac{1}{2} f(r)\left(\phi^{\prime}\right)^{2}+\frac{1}{2} m^{2} \phi^{2} \\
P_{r}=\frac{1}{2} f(r)\left(\phi^{\prime}\right)^{2}+\frac{1}{2} m^{2} \phi^{2}
\end{gathered}
$$

Field equation of motion is :

$$
\frac{1}{r^{2}} \frac{d\left(r^{2} f(r) \frac{d \phi}{d r}\right)}{d r}+\mu^{2} \phi=0
$$

Where :

$$
\mu^{2}=\xi R+m^{2}
$$

Also we define index $w$ as :

$$
w=\frac{P}{\rho}
$$

In this equation pressure is radial component of energy-momentum tensor. We choose radial pressure to preserve isotropy of our spacetime and not to disturb the matter distribution enclosed in our model.

\section{ASYMPTOTIC SOLUTIONS}

In equation (12) we substitute $\phi(r)$ by $\psi(r)$ :

$$
\phi(r)=\frac{\psi(r)}{r \sqrt{f(r)}},
$$


Which gives :

$$
\frac{d^{2} \psi(r)}{d r^{2}}+v(r) \psi(r)=0
$$

Where :

$$
v(r)=\frac{1}{4}\left(\frac{f(r)^{\prime}}{f(r)}\right)^{2}-\frac{f(r)^{\prime}}{r f(r)}-\frac{1}{2} \frac{f(r)^{\prime \prime}}{f(r)}+\frac{\mu^{2}}{f(r)}
$$

We mention here that there is no orthogonal basis functions for this equation and we can not construct an exact solution for this equation and one can show that only there is an asymptotic semi-analytic solution for it. In fact if we use series method we take :

$$
\psi(r)=\sum_{n=6}^{\infty} b_{n} r^{n}
$$

Where recursion relation for series coefficients is :

$$
\begin{aligned}
& 4 b_{n}(x+y)^{2}(n-1 / 2)^{2} y^{2} x^{2}- \\
& 8 b_{n-1} x y(n-1)(n-2)(x+y)\left(y^{2}+x y+x^{2}\right) \\
& +4 b_{n-2}(n-2)(n-3)\left(y^{2}+x y+x^{2}\right)^{2} \\
& -4 b_{n-3} x y\left(\mu^{2} a^{2}-2 n^{2}+14 n-20\right)(x+y) \\
& +4 b_{n-4}\left(y^{2}+x y+x^{2}\right)\left(-2 n^{2}+18 n-37+\mu^{2} a^{2}\right) \\
& +b_{n-6}\left(160+4 n^{2}-52 n-4 \mu^{2} a^{2}\right)=0, n \geqslant 6
\end{aligned}
$$

There is no general method to solving this equation and we can use from an iteration method based on this equation which we do not present it here because it is difficult to represent coefficients in terms of polynomials. But we can prove that since the usual convergence tests for infinite series is valid in the interval of $x<r<y$ the series solution (18) is converged. In fact if we denote coefficients of

$$
b_{n-k}, k=0, \ldots 6
$$

by $c_{k}$ then we must show that

$$
\lim _{n \rightarrow \infty} \frac{b_{n+1}}{b_{n}}=\mathfrak{L}<1
$$

This inequality must be valid for all terms of $b_{n}$ thus we must have

$$
\lim _{n \rightarrow \infty} \frac{b_{n-k}}{b_{n-k+1}}=\mathfrak{L}<1, \forall k=2,3,4,6
$$

But it is obvious that:

$$
\lim _{n \rightarrow \infty} \frac{b_{n+1}}{b_{n}}<-\frac{c_{1}+c_{2}+c_{3}+c_{4}+c_{6}}{c_{0}}
$$


We define a new function

$$
F=-\frac{c_{1}+c_{2}+c_{3}+c_{4}+c_{6}}{c_{0}}
$$

. In terms of $x, y$ explicitly we can write that :

$$
F-1=-\frac{(y-1)^{2}(x-1)^{2}(y+x+1)^{2}}{(y+x)^{2}(y)^{2}(x)^{2}}<0
$$

Which is hold for all values of $\mathrm{x}, \mathrm{y}$ also if $y>x$. Then series (18) is converged. Now we take one simple approach to the problem. The best approach is using asymptotic expansion of solutions near physical horizons $r_{c}, r_{+}$and considering in details the phase transition . For simplicity we denote horizons $r_{c}$ by $x$ and $r_{+}$by $y$. Then by simple algebra we have :

$$
f(r)=\frac{-(r-x)(r-y)(r+x+y)}{a^{2} r}
$$

By expanding $v(r)$ near $r=x$ we obtain :

$$
v(r) \simeq \frac{1}{4}(r-x)^{-2}+O\left[(r-x)^{-1}\right]
$$

Then near $r=x$ asymptotic solution for (16) is :

$$
\psi(r)=\sqrt{r-x}\left(c_{1}+c_{2} \ln (r-x)\right)
$$

This solution vanishes identically near $r=x$ and satisfy Dirichlet-BoundaryCondition(D.B.C) on the surface $r=x$. By rewriting this solution in the form of $\phi(r)$ we obtain :

$$
\phi(r)=\frac{\sqrt{r-x}\left(c_{1}+c_{2} \ln (r-x)\right)}{r \sqrt{f(r)}}
$$

Thus near $r=x$, the expansion of E.O.S (14) up to 2'nd order is :

$$
w \simeq 0
$$

Similarly near $r=y$ :

$$
v(r) \simeq \frac{1}{4}(r-y)^{-2}+O\left[(r-y)^{-1}\right]
$$

With the same D.B.C we've :

$$
\psi(r)=\sqrt{r-y}\left(c_{1}+c_{2} \ln (r-y)\right)
$$

Thus near $r=y$, E.O.S up to 2'nd order is :

$$
w \simeq 0
$$


Thus our scalar field near both horizons behaves like a dust with $w=0$.

On the other hand spacetime which close to the B.H. is Schwarzschild-like and far away from $y$ we have de Sitter spacetime with no singularity therefore we could preserve the form (1) .Near both Schwarzschild or de Sitter horizons(i.e; $r=2 M \& r=a$ ) solutions for (16) are derived from a simple Airy function with a simple D.B.C as mentioned before that is :

$$
\begin{gathered}
\psi(r)=-\operatorname{Airy} A\left(\frac{v_{0}+v_{1}(r-h)}{-v_{1}^{2 / 3}}\right) \operatorname{Airy} B\left(\frac{-v_{0}}{v_{1}^{2 / 3}}\right) \\
+\operatorname{Airy} B\left(\frac{v_{0}+v_{1}(r-h)}{-v_{1}^{2 / 3}}\right) \operatorname{Airy} A\left(\frac{-v_{0}}{v_{1}^{2 / 3}}\right)
\end{gathered}
$$

Where $h$ can be $2 M$ or $a, v_{0}=v(r=h)$ and $v_{1}=v^{\prime}(r=h)$. Finally near this region we've :

$$
w \simeq\left(\frac{1}{4 \xi-1}\right)+O\left[(r-h)^{2}\right]
$$

Which for a minimally coupled scalar field our matter behaves like an exotic matter with a similar equation of state as Dark energy in the cosmos does. If we focused only on the negative pressure matters we obtain a new fascinated constraint on the value of coupling as :

$$
\xi<\frac{1}{4}
$$

Which both interesting cases (minimally and conformally coupled)belongs to this interval. We mention here that this idea, scalar fields can change a phase from dust to negative pressure matter is an anstaz and we can not deduce a full complete description of the mechanism which is hidden behind this event.

\section{SOME FURTHER PHYSICAL NOTES}

Why we take scalar field responsible for phase transition? This is an anstaz for simplicity. We should mention here that there is no exact solution for a spherically symmetric solution for field equations with a massive non minimally coupled source . Thus from a mathematically point of view, one can take scalar field as a perturbation to our spherically symmetric metric in four dimensions. Also it can be shown that a minimally coupled -massless -scalar field in any spherically symmetric spacetime behaves like a fluid with equation of state $P=-\rho$. That is dark energy!. Thus any minimally coupled scalar field in a spherically symmetric background behaves like a pure dark energy. One can show that there is no phase transition from dark energy to dark matter in this case. Therefore phase transition is caused just for none minimally coupled massive scalar field. Therefor the local E.O.S. doesn't include mass $\left(\mathrm{m}^{2}\right)$ to first order so even having a coupled massless scalar field could cause this transition.

\section{SUMMARY}

In this work first we derived some asymptotic solutions near both cosmological and event horizons of SdS and showed that our field behaves as a dust (dark matter) near these 
horizons. We showed that far a way from them our field behaves like a matter with negative pressure and this limited ourselves to some constraints about the coupling constant $\xi$.

\section{ACKNOWLEDGEMENT}

This work was supported by University of Tehran (Iran)

* Electronic address: davood.momeni@kiau.ac.ir

$\dagger$ Electronic address: azadi@phymail.ut.ac.ir

[1] Supernova Search Team (A.G. Riess et al.), Astrophys. J. 607 (2004),665.

[2] R.R. Caldwell, Phys. Lett. B 545 (2002), 23

A.A. Coley, S.Hervik, W.C. Lim, gr-qc/0512085.

[3] M.C. Bento, O. Bertolami, A.A. Sen, Phys. Rev. D 66 (2002), 043507

Phys. Lett. B 575 (2003), 172

N. Bilic, G.B. Tupper , R.D.Viollier, Phys. Lett. B 535 (2002), 17

A. Kamenshchik, U.Moschella, V. Pasquier, Phys. Lett. B 511 (2001) 265

T.Multamaki, M. Manera, E.Gazta?naga, Phys. Rev. D 69 (2004), 023004.

[4] B. Ratra, P.J.E. Peebles, Phys. Rev. D 37 (2000), 3406

E.Elizalde, S. Nojiri, S.D. Odintsov, Phys. Rev. D 70 (2004), 043539

H. Wei, R.-G. Cai, Phys. Rev. D 72 (2005), 123507

H. Wei, R.-G. Cai , D.-F. Zeng, Class. Quant. Grav. 22 (2005),3189

D.F. Mota , C. van de Bruck, Astron. Astrophys. 421 (2004), 71

F. Perrotta , C. Baccigalupi, Phys. Rev. D 65 (2002), 123505

M. Alcubierre, F.S. Guzman, T. Matos, D. Nunez, L.A. Urena-Lopez, P. Wiederhold, Class. Quant. Grav. 19 (2002), 5017

W. Hu, R. Barkana , A. Gruzinov, Phys. Rev. Lett. 85 (2000), 1158

[5] T. Padmanabhan, T.R. Choudhury, Phys. Rev. D 66 (2002), 081301(R).

[6] Otto Begmann and Roy Leipnik.Phys.Rev.Vol 107.4.1957

[7] H.A.Buchdahl.Phys.Rev.Vol 115.Num 5.1959

Max Wyman.Phys.Rev.D.Vol 24.4.1981

Basilis C.Xanthopoulos and Thomas Zannias.Phys.Rev.D.Vol 40.8,1989

[8] N.D.Birrell, P.C.Davis, Quantum fields in curved space, Cambridge U.P.,1989.

[9] R.A. Konoplya, A. Zhidenko, Nucl.Phys.B777:182,2007.

[10] R.A. Konoplya, A. Zhidenko, JHEP 0406:037,2004. 